\title{
Pressure Overload Selectively Increases n-3 PUFA in Myocardial Phospholipids During Early Postnatal Period
}

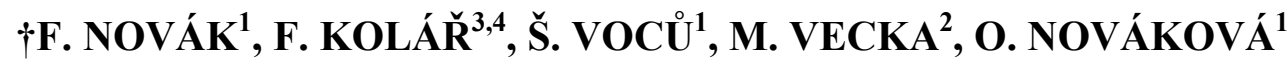 \\ ${ }^{1}$ Department of Cell Biology, Faculty of Science, Charles University, Prague, Czech Republic, \\ ${ }^{2}$ First Faculty of Medicine, Charles University, Prague, Czech Republic, ${ }^{3}$ Centre for Cardiovascular \\ Research, Prague, Czech Republic, ${ }^{4}$ Institute of Physiology, Academy of Sciences of the Czech \\ Republic, Prague, Czech Republic
}

Received June 2, 2012

Accepted June 4, 2012

$\dagger$ Deceased June 11, 2012

\begin{abstract}
Summary
Increasing hemodynamic load during early postnatal development leads to rapid growth of the left ventricular (LV) myocardium, which is associated with membrane phospholipid $(P L)$ remodeling characterized by $n-3$ polyunsaturated fatty acids (PUFA) accumulation. The aim of this study was to examine the influence of additional workload imposed early after birth when ventricular myocytes are still able to proliferate. Male Wistar rats were subjected to abdominal aortic constriction (AC) at postnatal day 2. Concentrations of PL and their fatty acid (FA) profiles in the LV were analyzed in $\mathrm{AC}$, sham-operated (SO) and intact animals on postnatal days 2 (intact only), 5 and 10 . AC resulted in LV enlargement by $22 \%$ and $67 \%$ at days 5 and 10, respectively, compared with age-matched SO littermates. Concentrations of phosphatidylcholine, cardiolipin, phosphatidylinositol, phosphatidylethanolamine, phosphatidylserine and sphingomyelin decreased in $\mathrm{AC}$ myocardium, albeit with different time course and extent. The main effect of $A C$ on $F A$ remodeling consisted in the accumulation of $n-3$ PUFA in PL. The most striking effect of AC on FA composition was observed in phosphatidylinositol and cardiolipin. We conclude that excess workload imposed by $\mathrm{AC}$ inhibited the normal postnatal increase of PL concentration while further potentiating the accumulation of n-3 PUFA as an adaptive response of the developing myocardium to accelerated growth.
\end{abstract}

\section{Key words}

Phospholipids • n-3 PUFA • Myocardium • Postnatal development - Pressure overload $\bullet$ Rat

\section{Corresponding author}

O. Nováková, Department of Cell Biology, Faculty of Science,
Charles University, Viničná 7, 12800 Prague 2, Czech Republic. Fax: +420 221951761. E-mail: olnov@natur.cuni.cz

\section{Introduction}

In early postnatal life, the developing mammalian heart must cope with increasing hemodynamic load, which is associated with rapid growth of the left ventricle (LV). While this period is one of continuing mitotic proliferation (hyperplasia), in most mammals, ventricular myocytes lose their capability for division shortly after birth and further growth of the heart is considered to occur due to their hypertrophy along with the hyperplasia and hypertrophy of other cell types (Clubb and Bishop 1984). It has been shown that the switch from hyperplastic to hypertrophic growth of rat ventricular myocytes occurs as early as after the third postnatal day (Li et al. 1996). Rapid heart growth during the first postnatal week is accompanied by metabolic, morphological and functional remodeling of myocardial tissue (Girard et al. 1992, Oštádal et al. 1999). Membrane structures are among the main targets of this developmental process, including both their protein (Novotný et al. 1999, Hamplová et al. 2003) and lipid components (Novák et al. 2006). As the main membrane lipids, phospholipids (PL) create the environment for membrane proteins. The functions of these proteins depend on the proportions of individual PL species and/or on their fatty acid (FA) compositions.

Numerous experimental results obtained mostly from isolated cardiomyocytes indicate that hydrolytic 
products derived from membrane PL by the action of phospholipases play an important role as second messengers in signal transduction involved in stimulating cell growth and proliferation (Dorn and Force 2005, Tappia 2007). In view of these observations, the remodeling of membrane PL induced by pathological load superimposed on physiological growth of the cardiomyocytes during early ontogeny may influence the postnatal structural and functional characteristics of the developing heart. In this study, we used a model of pressure overload induced by aortic constriction (AC) in neonatal rats at postnatal day 2 as described earlier (Koláŕ et al. 1998). The concentration of individual PL species and their FA profiles were analyzed at days 5 and 10 . This model allowed us to follow the adaptive response of membrane lipid components in myocardium which was still in the proliferative phase of ventricular myocyte growth (Clubb and Bishop 1984, Sedmera et al. 2003).

\section{Methods}

Newborn male Wistar rats were obtained from the animal care facility of the Institute of Physiology in Prague, Czech Republic. Their number per litter was kept at eight throughout the weaning period. Nursing mothers were fed a vitamin-enriched, low-fat (3.5\% by weight) standard diet (Velaz, Czech Republic). The diet contained the following FA (in mol \%), 1.5 of 14:0, 19.2 of $16: 0,2.2$ of $16: 1 \mathrm{n}-7,5.5$ of $18: 0,25.4$ of $18: 1 \mathrm{n}-9,1.9$ of $18: 1 \mathrm{n}-7,38.7$ of $18: 2 \mathrm{n}-6$, and 4.2 of 18:3n-3. Pressure overload was induced by constriction of the abdominal aorta (AC) in randomly selected animals in each litter. This intervention was performed in 2-day-old rats under light ether anesthesia as described earlier (Koláŕ et al. 1998). The abdominal cavity was opened by a small incision from the left dorsolateral side, and the aorta was exposed in the subdiaphragmatic suprarenal region. A silk ligature was tied around the aorta and a hypodermic needle of $0.25 \mathrm{~mm}$ outer diameter. The needle served as a template, and after its subsequent removal the aorta remained constricted to the diameter of the template. The incision was sutured and smeared with $1 \%$ iodcollodium. Two types of controls were used for this study: intact littermates (intact) and sham-operated pups (SO) of the same age for which the identical procedure was carried out but without placement of the ligature. The animals were sacrificed by decapitation on days 2 (intact only), 5 and 10 (i.e., 3 and 8 days after the surgery). The study was conducted in accordance with the Guide for the Care and Use of Laboratory Animals published by the US National Institutes of Health (NIH publication no. 85-23, revised 1996). The experimental protocol was approved by the Animal Care and Use Committee of the Institute of Physiology, Academy of Sciences of the Czech Republic.

The hearts were dissected free of atrial tissue and large blood vessels and the right ventricular (RV) free wall and septum were separated from the LV. All parts were rinsed in cold $\left(5^{\circ} \mathrm{C}\right)$ saline, weighed separately, and the LV was frozen in liquid nitrogen and stored there until use. Frozen LV myocardium was pulverized and homogenized. PL were extracted in three consecutive steps according to the method of Folch et al. (1957). Phosphatidylcholine (PC), phosphatidylethanolamine (PE), cardiolipin (CL), phosphatidylinositol (PI), phosphatidylserine (PS) and sphingomyelin (SM) were separated by twodimensional thin-layer chromatography on plates with Silica Gel H $(0.5 \mathrm{~mm})$, the individual PL spots were detected by iodine vapor, then scraped out and analyzed for phosphorus (Rouser et al. 1970). For FA analysis, the spots of individual PL were observed under UV light after staining with $0.005 \%$ 2,7-dichlorofluorescein in methanol, scraped out and stored in nitrogen atmosphere at $-20^{\circ} \mathrm{C}$ until the next day, at which time methyl esters were prepared. For FA methyl ester preparation, sodium methanolate was added to tubes with silica gel and tubes were then incubated for $60 \mathrm{~min}$ at room temperature in darkness. Methyl esters were extracted with hexane; the extracts were evaporated under a stream of nitrogen and stored at $-20{ }^{\circ} \mathrm{C}$. FA methyl esters were separated by CP 438 A gas chromatograph (Chrompack, Middelburg, The Netherlands) using a CP WAX 52 CB middle polar column $(25 \mathrm{~m} \times 0.25 \mathrm{~mm}$ i.d. $)$. The oven temperature was programmed from 145 to $230{ }^{\circ} \mathrm{C}$ at $2{ }^{\circ} \mathrm{C} / \mathrm{min}$. Hydrogen was used as carrier gas. FA were identified using a mixture of FA methyl ester standards (Tvrzická et al. 2002). All chemicals were purchased from Sigma unless otherwise indicated.

\section{Statistical analysis}

All results are expressed as means \pm S.E.M. The statistical significance of differences between groups was determined by one-way ANOVA and subsequent Newman-Keuls test. Differences were considered significant when $P<0.05$. 
Table 1. Effects of aortic constriction on body and heart weight parameters.

\begin{tabular}{lccccccc}
\hline & Day 2 & \multicolumn{3}{c}{ Day 5 } & \multicolumn{2}{c}{ Day 10 } \\
\cline { 2 - 8 } & Intact & Intact & SO & AC & Intact & SO & AC \\
\hline$B W(g)$ & $6.08 \pm 0.15$ & $13.30 \pm 0.35$ & $12.60 \pm 0.36$ & $12.80 \pm 0.31$ & $24.50 \pm 0.70$ & $24.90 \pm 0.54$ & $24.30 \pm 0.83$ \\
$H W(m g)$ & $37.30 \pm 0.87$ & $64.30 \pm 1.27$ & $59.20 \pm 1.30^{\dagger}$ & $69.10 \pm 1.92^{\ddagger}$ & $99.10 \pm 2.31$ & $99.60 \pm 2.53$ & $160.90 \pm 16.4^{\ddagger \$}$ \\
$L V(m g)$ & $19.90 \pm 0.59$ & $32.30 \pm 0.90$ & $29.10 \pm 1.00^{\dagger}$ & $36.30 \pm 1.24^{\ddagger}$ & $54.20 \pm 1.59$ & $53.30 \pm 1.54$ & $95.80 \pm 9.98^{\ddagger \$}$ \\
$R V(m g)$ & $10.10 \pm 0.29$ & $16.60 \pm 0.49$ & $15.90 \pm 0.46$ & $16.30 \pm 0.43$ & $24.20 \pm 0.71$ & $24.20 \pm 0.84$ & $34.80 \pm 3.47^{\ddagger \$}$ \\
$S(m g)$ & $7.30 \pm 0.24$ & $15.30 \pm 0.45$ & $14.30 \pm 0.33^{\dagger}$ & $16.50 \pm 0.72^{\ddagger}$ & $20.80 \pm 0.74$ & $22.10 \pm 0.99$ & $30.30 \pm 3.14^{\ddagger \$}$ \\
$H W / B W(\%)$ & $0.55 \pm 0.01$ & $0.49 \pm 0.01$ & $0.47 \pm 0.01$ & $0.54 \pm 0.01^{\ddagger}$ & $0.41 \pm 0.01$ & $0.40 \pm 0.01$ & $0.60 \pm 0.09^{\ddagger}$ \\
$L V / B W(\%)$ & $0.29 \pm 0.01$ & $0.24 \pm 0.01$ & $0.23 \pm 0.01$ & $0.28 \pm 0.01^{\ddagger}$ & $0.22 \pm 0.01$ & $0.21 \pm 0.01$ & $0.35 \pm 0.05^{\ddagger \$}$ \\
$R V / B W(\%)$ & $0.15 \pm 0.01$ & $0.13 \pm 0.01$ & $0.13 \pm 0.01$ & $0.13 \pm 0.01$ & $0.10 \pm 0.01$ & $0.10 \pm 0.01$ & $0.13 \pm 0.02$ \\
$R V / L V$ & $0.53 \pm 0.02$ & $0.52 \pm 0.02$ & $0.56 \pm 0.02$ & $0.46 \pm 0.02^{\ddagger}$ & $0.46 \pm 0.02$ & $0.46 \pm 0.02$ & $0.36 \pm 0.01^{\ddagger \$}$ \\
\hline
\end{tabular}

SO - sham-operation; AC - aortic constriction; BW - body weight; HW - heart weight; LV - left ventricle; RV - right ventricle; $\mathrm{S}$ - septum. Values are mean \pm S.E.M. from 20 experiments in each group. ${ }^{\dagger} \mathrm{P}<0.05$ vs. age-matched intact; ${ }^{\ddagger} \mathrm{P}<0.05$ vs. age-matched SO; ${ }^{\$} \mathrm{P}<0.05$ vs. AC at day 5 .

\section{Results}

\section{Weight parameters}

Body weight was significantly altered by neither SO nor AC in any age group. SO caused moderate heart and $\mathrm{LV}$ growth retardation at day 5, but these effects waned by day 10. AC led to the LV's enlargement, as evident from $22 \%$ increase in the LV/BW ratio by day 5 and $67 \%$ increase by day 10 compared with age-matched SO littermates. While AC slightly increased RV weight, the $\mathrm{RV} / \mathrm{BW}$ ratio was not significantly influenced (Table 1).

\section{Concentration of phospholipids}

Table 2 presents the developmental changes in the concentration of individual PL species in LV of intact animals between days 2 and 10 and the effects caused by SO and AC. The normal physiological increment of total PL in intact pups was $17 \%$ between days 2 and 5. During the period, PC and PE increased by $17 \%$, CL by $33 \%$ and PI by $20 \%$. There was no further significant increase in the concentration of any PL between days 5 and 10. The concentrations of PS and SM remained at unchanged levels during the entirety of the investigated period. The effect of SO on PL concentration was observed only at day 10, when total PL concentration increased by $10 \%$ compared with intact animals due to slight increases in all PL (significant only in the case of PE). AC decreased the concentration of total PL by $12 \%$ and $14 \%$ at days 5 and 10, respectively, compared with age-matched $\mathrm{SO}$ littermates. This effect was due to decreases of PC, PI and CL by $11 \%, 16 \%$ and $17 \%$, respectively, at day 5 and of PC, PE, CL, PS and SM by $12 \%, 15 \%, 25 \%, 24 \%$ and
$17 \%$, respectively, at day 10 . No significant difference in the individual PL concentrations was observed between days 5 and 10, except for increase of PI by $11 \%$ and decrease of PS by $18 \%$ at day 10 .

\section{Fatty acid composition of phospholipids}

Figure 1 shows the proportions of FA classes in myocardial PL of intact, SO and AC animals. Saturated FA (SFA), monounsaturated FA (MUFA), n-6 and n-3 polyunsaturated FA (PUFA) in PC, PI, and CL at postnatal days 5 and 10 are expressed as the percentage of values for the intact 2-day-old group, which are presented in Table 3. FA that were mainly responsible for the changes induced by $\mathrm{AC}$ in PL are also shown in Figure 1. In intact hearts, the n-3 PUFA proportion in $\mathrm{PC}$ and $\mathrm{CL}$ gradually increased while that of MUFA declined. The increase of n3 PUFA in PI was accompanied by the decrease in both MUFA and n-6 PUFA.

AC slightly increased the n-3 PUFA and MUFA proportions at day 10 in PC. The most pronounced effect of AC was observed in PI where the proportions of MUFA and n-3 PUFA increased while that of n-6 PUFA declined. In PI, docosapentaenoic acid (22:5n-3, DPA) and docosahexaenoic acid (22:6n-3, DHA) were responsible for the elevation of n-3 PUFA, oleic acid (18:1n-9, OA) was the main contributor to the increase of MUFA, and the decrease of n-6 PUFA was caused by arachidonic acid (20:4n-6, AA). Concerning the effect of AC on CL, the increase in n-3 PUFA was present already at day 5 and more pronounced at day 10, offset by a decrease in MUFA. The increases of 22:5n-3 and 22:6n-3 and the decreases of 18:1n-9 and linoleic acid (18:2n-6, LA) were mainly responsible for these effects. 
Table 2. Effect of aortic constriction on myocardial concentration of phospholipids ( $\mu \mathrm{mol}$ P. $\mathrm{g}^{-1}$ wet weight).

\begin{tabular}{lccccccc}
\hline & \multicolumn{5}{c}{ Day 2 } & \multicolumn{1}{c}{ Day 5 } & \multicolumn{3}{c}{ Day 10 } \\
\cline { 2 - 8 } & \multicolumn{1}{c}{ Intact } & Intact & \multicolumn{1}{c}{ SO } & AC & Intact & SO & AC \\
\hline$P C$ & $8.47 \pm 0.30$ & $9.92 \pm 0.14$ & $10.56 \pm 0.40$ & $9.37 \pm 0.30^{\ddagger}$ & $10.16 \pm 0.38$ & $11.03 \pm 0.29$ & $9.70 \pm 0.37^{\ddagger}$ \\
$P E$ & $6.07 \pm 0.18$ & $7.12 \pm 0.15$ & $7.51 \pm 0.20$ & $6.85 \pm 0.21$ & $7.45 \pm 0.36$ & $8.25 \pm 0.11^{\dagger}$ & $7.04 \pm 0.13^{\ddagger}$ \\
$C L$ & $1.22 \pm 0.09$ & $1.62 \pm 0.07$ & $1.86 \pm 0.11$ & $1.54 \pm 0.07^{\ddagger}$ & $1.82 \pm 0.08$ & $2.02 \pm 0.10$ & $1.52 \pm 0.09^{\ddagger}$ \\
$P I$ & $1.06 \pm 0.03$ & $1.27 \pm 0.03$ & $1.39 \pm 0.03$ & $1.17 \pm 0.03^{\ddagger}$ & $1.18 \pm 0.05$ & $1.32 \pm 0.04$ & $1.30 \pm 0.02^{\$}$ \\
$P S$ & $0.89 \pm 0.02$ & $0.94 \pm 0.02$ & $1.03 \pm 0.05$ & $0.90 \pm 0.04$ & $0.90 \pm 0.07$ & $0.97 \pm 0.05$ & $0.74 \pm 0.04^{\ddagger}$ \\
$S M$ & $0.80 \pm 0.03$ & $0.83 \pm 0.03$ & $0.83 \pm 0.05$ & $0.74 \pm 0.04$ & $0.86 \pm 0.04$ & $0.94 \pm 0.02$ & $0.78 \pm 0.04^{\ddagger}$ \\
$\Sigma P L$ & $18.57 \pm 0.31$ & $21.77 \pm 0.34$ & $23.23 \pm 0.70$ & $20.53 \pm 0.66^{\ddagger}$ & $22.43 \pm 0.71$ & $24.57 \pm 0.34^{\dagger}$ & $21.12 \pm 0.56^{\ddagger}$ \\
\hline
\end{tabular}

SO - sham-operation; AC - aortic constriction; PL - phospholipids; PC - phosphatidylcholine; PE - phosphatidylethanolamine; $\mathrm{CL}$ - cardiolipin; PI - phosphatidylinositol; PS - phosphatidylserine; SM - sphingomyelin. Values are mean \pm S.E.M. from 6 experiments in each group. ${ }^{\dagger} \mathrm{P}<0.05$ vs. age-matched intact; ${ }^{\ddagger} \mathrm{P}<0.05$ vs. age-matched $\mathrm{SO} ;{ }^{\$} \mathrm{P}<0.05$ vs. AC at day 5.

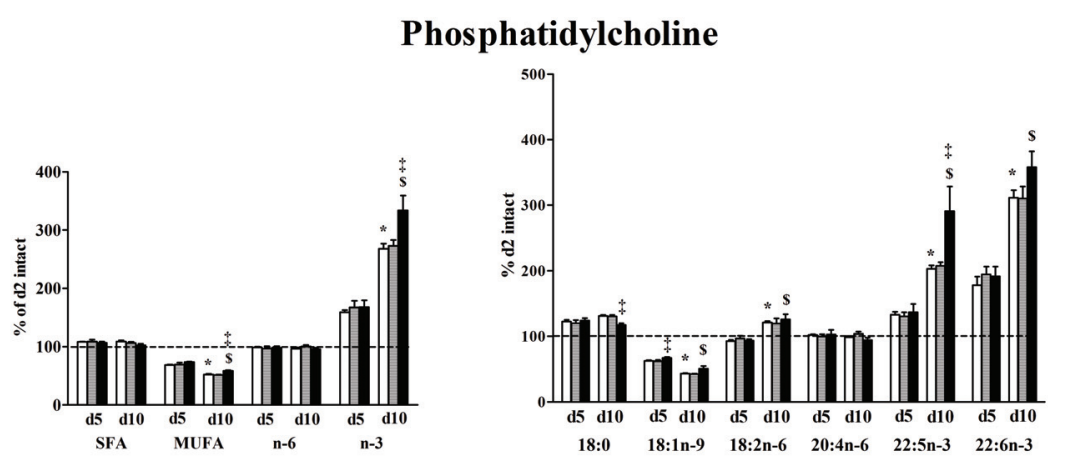

Phosphatidylinositol
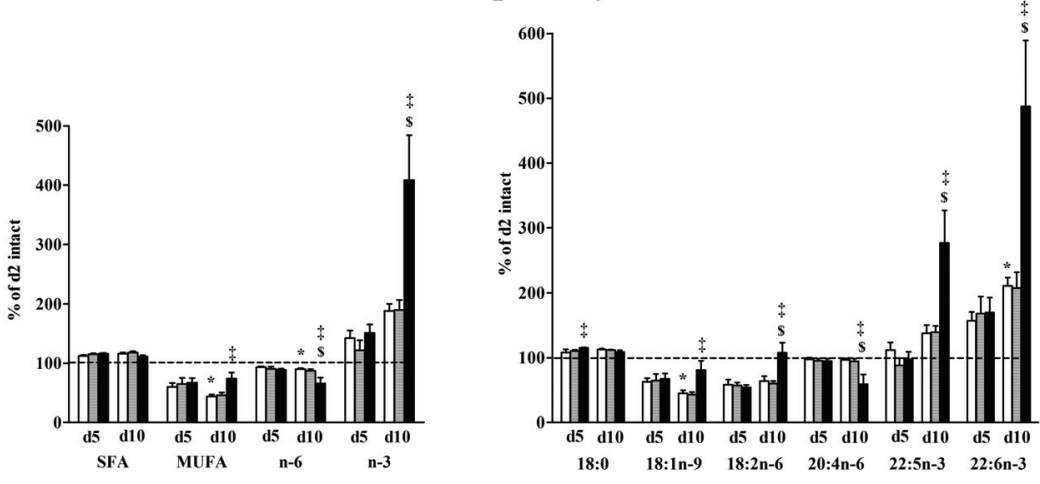

\section{Cardiolipin}
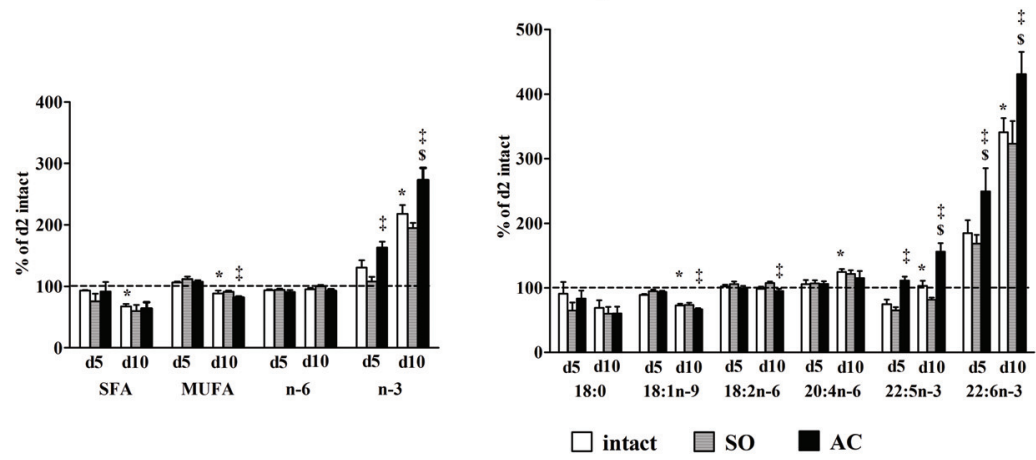

Fig. 1. Proportions of fatty acid (FA) classes and main $F A$ in selected phospholipids of the left ventricle of intact, sham-operated (SO) and aorta-constricted (AC) rats at postnatal days 5 and 10 (i.e., 3 and 8 days after $A C$ ), expressed as percentages of values for 2-day-old intact controls. SFA - sum of saturated FA; MUFA sum of monounsaturated $F A ; n-6$ - sum of $n-6$ polyunsaturated FA (PUFA); $n-3$ - sum of $n-3$ PUFA; $18: 0$ - stearic acid; $18: 1 n-9$ - oleic acid; 18:2n-6 - linoleic acid; 20:4n-6 - arachidonic acid; $22: 5 n-3$ - docosapentaenoic acid; 22:6n-3 - docosahexaenoic acid. Values are means \pm S.E.M. from 6 experiments in each group. ${ }^{*} \mathrm{P}<0.05$ vs. intact at day $5 ;{ }^{\ddagger} \mathrm{P}<0.05$ vs. agematched SO; ${ }^{\$} \mathrm{P}<0.05$ vs. AC at day 5 . Shown are only those FA that were mainly responsible for AC-induced effects. 
Table 3. Fatty acid (FA) composition (mol \%) of myocardial phospholipids in 2-day-old intact rats.

\begin{tabular}{lccccc}
\hline FA & PC & PE & CL & PI & PS \\
\hline$S F A$ & $45.78 \pm 2.12$ & $30.55 \pm 0.99$ & $9.00 \pm 1.12$ & $39.54 \pm 0.60$ & $52.34 \pm 5.80$ \\
$18: 0$ & $18.10 \pm 0.66$ & $23.51 \pm 0.27$ & $3.71 \pm 0.66$ & $34.16 \pm 1.18$ & $45.66 \pm 5.20$ \\
MUFA & $17.76 \pm 0.45$ & $8.73 \pm 0.39$ & $33.25 \pm 0.87$ & $9.88 \pm 0.60$ & $10.27 \pm 1.01$ \\
$18: 1 n-9$ & $11.26 \pm 0.32$ & $5.83 \pm 0.24$ & $20.15 \pm 0.42$ & $6.50 \pm 0.34$ & $8.03 \pm 1.60$ \\
$n-6$ & $33.15 \pm 1.83$ & $48.63 \pm 0.96$ & $50.89 \pm 1.26$ & $46.24 \pm 0.67$ & $22.28 \pm 2.30$ \\
$18: 2 n-6$ & $3.17 \pm 0.13$ & $1.63 \pm 0.13$ & $29.59 \pm 1.11$ & $2.37 \pm 0.14$ & $1.85 \pm 0.31$ \\
$20: 4 n-6$ & $26.95 \pm 1.41$ & $38.14 \pm 0.78$ & $10.41 \pm 0.73$ & $38.97 \pm 1.19$ & $9.21 \pm 1.38$ \\
$n-3$ & $3.31 \pm 0.48$ & $12.08 \pm 0.80$ & $6.85 \pm 0.57$ & $4.40 \pm 0.50$ & $14.82 \pm 1.99$ \\
$22: 5 n-3$ & $1.33 \pm 0.19$ & $3.99 \pm 0.44$ & $2.47 \pm 0.47$ & $1.88 \pm 0.17$ & $4.92 \pm 0.81$ \\
$22: 6 n-3$ & $1.86 \pm 0.34$ & $7.87 \pm 0.45$ & $3.31 \pm 0.17$ & $2.53 \pm 0.37$ & $9.15 \pm 1.18$ \\
\hline
\end{tabular}

SO - sham-operation; AC - aortic constriction; PC - phosphatidylcholine; PE - phosphatidylethanolamine; $\mathrm{CL}$ - cardiolipin; PI - phosphatidylinositol; PS - phosphatidylserine; SFA - sum of saturated FA; MUFA - sum of monounsaturated FA; $n-6$ - sum of $n-6$ polyunsaturated FA (PUFA); n-3 - sum of n-3 PUFA; 18.0 - stearic acid; $18: 1 n-9$ - oleic acid; $18: 2 n-6$ - linoleic acid; $20: 4 n-6$ arachidonic acid; 22:5n-3 - docosapentaenoic acid; 22:6n-3 - docosahexaenoic acid. Values are mean \pm S.E.M. from 6 experiments in each group. Shown are only those FA that were mainly responsible for AC-induced effects.

\section{Phosphatidylethanolamine}

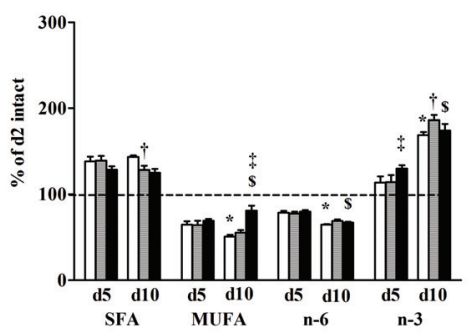

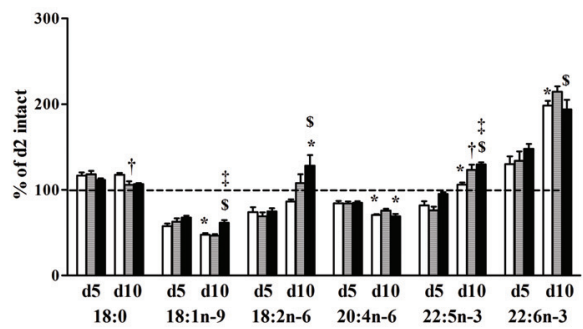

18:0 18:1n-9 18:2n-6 20:4n-6 22:5n-3 22:6n-3
Fig. 2. Proportions of fatty acid (FA) classes and main FA in aminophospholipids of left ventricle of intact, sham-operated (SO) and aorta-constricted (AC) rats at postnatal days 5 and 10 (i.e., 3 and 8 days after AC), expressed as a percentage of 2-day-old intact controls. ${ }^{\dagger} \mathrm{P}<0.05$ vs. agematched intact. See Figure 1 for further legend.

\section{Phosphatidylserine}
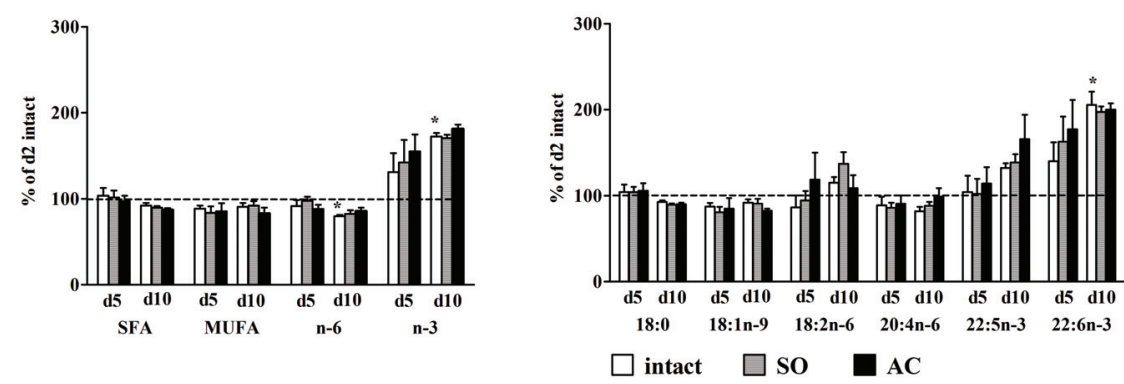

Figure 2 shows the relative proportions of FA classes in myocardial amino-phospholipids (PE and PS) in intact, SO and AC groups. Gradual developmental increase in n-3 PUFA in PE was accompanied by decrease of both MUFA and n-6 PUFA. Slight increase in n-3 PUFA and decrease in n-6 PUFA were observed in PS at day 10. PE was the only PL affected by SO, resulting in a slight increase in n-3 PUFA and decrease in
SFA at day 10. Concerning the effects of AC, increase in n-3 PUFA (due to 22:5n-3) was seen already at day 5, waning at day 10 , whereas a decreased proportion of MUFA was maintained until day 10. No effect of AC on FA composition was observed in PS.

Changes in the n-6/n-3 PUFA, 20:4n-6/18:2n-6 and 20:4n-6/22:6n-3 ratios induced by AC are shown in Table 4. AC did not affect these ratios in any PL at day 5, 
Table 4. Effects of aortic constriction on 20:4/18:2, 20:4/22:6 and n-6/n-3 PUFA ratios in myocardial phospholipids.

\begin{tabular}{|c|c|c|c|c|c|c|c|}
\hline & \multirow{2}{*}{$\frac{\text { Day } 2}{\text { Intact }}$} & \multicolumn{3}{|c|}{ Day 5} & \multicolumn{3}{|c|}{ Day 10} \\
\hline & & Intact & SO & $\mathbf{A C}$ & Intact & SO & $\mathbf{A C}$ \\
\hline \multicolumn{8}{|l|}{$P C$} \\
\hline $20: 4 / 18: 2$ & $8.50 \pm 0.51$ & $9.44 \pm 0.68$ & $8.75 \pm 0.45$ & $9.31 \pm 0.53$ & $6.92 \pm 0.15^{*}$ & $7.52 \pm 0.55$ & $6.47 \pm 0.52$ \\
\hline $20: 4 / 22: 6$ & $14.49 \pm 0.84$ & $8.29 \pm 0.49$ & $7.42 \pm 0.93$ & $7.77 \pm 0.63$ & $4.61 \pm 0.12^{*}$ & $4.92 \pm 0.32$ & $3.86 \pm 0.23^{\star \$}$ \\
\hline$n-6 / n-3$ & $10.02 \pm 1.25$ & $6.12 \pm 0.53$ & $5.78 \pm 0.49$ & $5.86 \pm 0.61$ & $3.62 \pm 0.07^{*}$ & $3.69 \pm 0.15$ & $2.91 \pm 0.17^{\$ \$}$ \\
\hline \multicolumn{8}{|l|}{$P E$} \\
\hline $20: 4 / 18: 2$ & $23.40 \pm 3.51$ & $26.77 \pm 2.65$ & $28.35 \pm 2.82$ & $26.59 \pm 1.90$ & $19.26 \pm 0.62^{*}$ & $17.04 \pm 1.37$ & $13.5 \pm 1.97^{\$}$ \\
\hline $20: 4 / 22: 6$ & $4.85 \pm 0.41$ & $3.16 \pm 0.18$ & $3.04 \pm 0.21$ & $2.79 \pm 0.17$ & $1.73 \pm 0.05^{*}$ & $1.72 \pm 0.07$ & $1.75 \pm 0.11$ \\
\hline$n-6 / n-3$ & $4.03 \pm 0.38$ & $2.78 \pm 0.16$ & $2.74 \pm 0.18$ & $2.48 \pm 0.15$ & $1.54 \pm 0.04^{*}$ & $1.49 \pm 0.06$ & $1.56 \pm 0.56^{\$}$ \\
\hline \multicolumn{8}{|l|}{$P S$} \\
\hline $20: 4 / 18: 2$ & $4.98 \pm 0.51$ & $5.11 \pm 0.65$ & $4.53 \pm 0.82$ & $3.80 \pm 0.90$ & $3.54 \pm 0.62$ & $3.20 \pm 0.37$ & $4.52 \pm 0.97$ \\
\hline $20: 4 / 22: 6$ & $1.01 \pm 0.41$ & $0.64 \pm 0.18$ & $0.53 \pm 0.21$ & $0.51 \pm 0.17$ & $0.40 \pm 0.05$ & $0.45 \pm 0.07$ & $0.50 \pm 0.11$ \\
\hline$n-6 / n-3$ & $1.50 \pm 0.18$ & $0.82 \pm 0.16$ & $0.82 \pm 0.18$ & $0.66 \pm 0.15$ & $0.69 \pm 0.04$ & $0.73 \pm 0.06$ & $0.71 \pm 0.06$ \\
\hline \multicolumn{8}{|l|}{$P I$} \\
\hline $20: 4 / 18: 2$ & $16.44 \pm 0.56$ & $27.54 \pm 0.68$ & $27.50 \pm 0.65$ & $28.87 \pm 0.53$ & $26.18 \pm 2.87$ & $26.56 \pm 2.70$ & $10.06 \pm 3.08^{\star s}$ \\
\hline $20: 4 / 22: 6$ & $15.40 \pm 0.87$ & $9.55 \pm 0.49$ & $8.74 \pm 0.53$ & $8.59 \pm 0.63$ & $7.19 \pm 0.58$ & $7.47 \pm 1.03$ & $2.59 \pm 1.07^{\$ \$}$ \\
\hline$n-6 / n-3$ & $10.51 \pm 0.81$ & $6.89 \pm 0.50$ & $6.73 \pm 0.49$ & $6.17 \pm 0.61$ & $5.12 \pm 0.41$ & $5.01 \pm 0.56$ & $2.11 \pm 0.67^{\ddagger \$}$ \\
\hline \multicolumn{8}{|l|}{$C L$} \\
\hline $20: 4 / 18: 2$ & $0.35 \pm 0.03$ & $0.36 \pm 0.04$ & $0.33 \pm 0.05$ & $0.38 \pm 0.06$ & $0.45 \pm 0.07^{*}$ & $0.40 \pm 0.04$ & $0.43 \pm 0.05$ \\
\hline $20: 4 / 22: 6$ & $3.15 \pm 0.34$ & $1.80 \pm 0.22$ & $1.80 \pm 0.21$ & $1.34 \pm 0.13$ & $1.15 \pm 0.11^{*}$ & $1.18 \pm 0.09$ & $0.85 \pm 0.08^{\$ \$}$ \\
\hline$n-6 / n-3$ & $7.43 \pm 0.45$ & $5.32 \pm 0.53$ & $6.49 \pm 0.49$ & $4.14 \pm 0.31^{\ddagger}$ & $3.24 \pm 0.14^{*}$ & $3.80 \pm 0.30$ & $2.58 \pm 0.24^{\ddagger \$}$ \\
\hline
\end{tabular}

SO - sham-operation; AC - aortic constriction; PC - phosphatidylcholine; PE - phosphatidylethanolamine; CL - cardiolipin; PI - phosphatidylinositol; PS - phosphatidylserine; $n-6$ - sum of $n-6$ polyunsaturated fatty acids (PUFA); $n-3$ - sum of $n-3$ PUFA; 18:2n-6 - linoleic acid; 20:4n-6 - arachidonic acid; 22:6n-3 - docosahexaenoic acid. Values are mean \pm S.E.M.

except for decline in the $n-6 / n-3$ PUFA ratio in CL. Significant decrease of n-6/n-3 PUFA and 20:4n-6/22:6n3 ratios occurred after $\mathrm{AC}$ at day 10 in PC, PI and CL. A decreased 20:4n-6/18:2n-6 ratio due to AC was observed also in PI at day 10. Unlike these PL, PS was resistant to $\mathrm{AC}$-induced changes.

\section{Discussion}

The major finding of the present study is that LV enlargement due to the excess workload imposed by AC early after birth was accompanied by decrease of membrane PL concentration compared with that in SO littermates. Moreover, AC further potentiated the normal developmental accumulation of n-3 PUFA in PL. Importantly, the individual PL FA profiles were selectively affected by AC.

Concerning the physiological development of PL composition, we confirmed our previous observation of marked increase in total PL concentration during the early postnatal period (by $32 \%$ between days 2 and 10). This suggests rapid proliferation of membrane structures - predominantly mitochondrial - as indicated by $49 \%$ increase in CL (Novák et al. 2006). Here, we show that the normal postnatal development of membranes, reflected by the increasing PL concentration, was inhibited by pressure overload selectively for each PL species. While the developmental increments of PC, PI and $\mathrm{CL}$ were attenuated by $\mathrm{AC}$ already at day 5, the decline of PE, PS and SM was observed only at day 10. The $31 \%$ decrease of CL between days 2 and 5 suggests that mitochondria were the main membrane structures affected by AC just in the most critical and stressful period characterized by rapid growth of the LV due to increasing hemodynamic load (Ošt’ádal et al. 1999). Impaired development of mitochondria in neonatal AC hearts had been previously documented by quantitative electron microscopy (Page and McCallister 1973).

After AC, the PI concentration declined at day 5, but, in contrast with other PL, it returned to the control 
level at day 10. This suggests a difference in regulation of the PI biosynthesis/degradation process that probably is due to its specific role in signal transduction pathways. PI is the most abundant phosphoinositide of the ventricular myocardium. It serves as a precursor for more phosphorylated phosphoinositides, generating inositol phosphate and diacylglycerols after phospholipase $\mathrm{C}$ activation. PC also plays an important role in signaling as a precursor of diacylglycerols and AA via the action of phospholipases $\mathrm{D}$ and $\mathrm{A} 2$, respectively. In these pathways, diacylglycerols activate protein kinase $\mathrm{C}$, a signaling molecule activating the expression of genes that are upregulated during myocardial hypertrophy (Dorn and Force 2005, Tappia 2007). Changes in PI are in agreement with our recent observation of a transient upregulation of $\mathrm{PKC} \alpha, \mathrm{PKC} \delta$ and $\mathrm{PKC} \varepsilon$ isoforms between postnatal days 2 and 5 in the same model of accelerated cardiac growth induced by AC (Hamplová et al. 2010). Furthermore, under the same conditions, Oliviero et al. (2000) have shown the transient increase of serum angiotensin II level already $2 \mathrm{~h}$ after $\mathrm{AC}$, followed by upregulation of both AT1 and AT2 receptors in the myocardium. Moreover, AT2 transcript levels closely correlated with the left ventricular mass in the AC group. It is well known that angiotensin II-mediated signal transduction is coupled with PL signaling molecules produced by stimulation of phospholipases (Dinh et al. 2001). The increase in angiotensin II level preceding the increase in its binding capacity and PKC activation/upregulation might suggest that this peptide is one of the triggers implicated in the phosphoinositides/PKC-mediated early response of neonatal myocardium to pressure overload. Nevertheless, other factors might also contribute to accelerated neonatal cardiac growth in response to pressure overload (Novák et al. 2009).

It should be mentioned that myocardium during this early developmental period responds to pressure overload differently than in the case of adult heart. When $\mathrm{AC}$ was performed at day 2, the increase of LV mass resulted first from the stimulated cellular hyperplasia followed later by enlargement of cardiomyocytes (Sedmera et al. 2003). In this experimental model, cardiomegaly was associated with capillary proliferation in proportion to the increase of ventricular mass and exhibited neither increased rates of apoptosis nor myocardial fibrosis characteristic for adult pressureoverloaded hearts (Kolář et al. 1998, Oliviero et al. 2000, Sedmera et al. 2003). In contrast to neonatal myocardium, rats subjected to $\mathrm{AC}$ in adulthood exhibited no decrease in total PL concentration in LV (Reibel et al. 1986). Nevertheless, the proportions of PC and PI increased at the expense of CL due to AC. It should be taken into account that the biosynthesis of protein and PL components predominates over their degradation during membrane maturation in the early postnatal period, whereas both processes are more in a dynamic equilibrium in the adult heart under physiological conditions (Girard et al. 1992, Nováková et al. 1994).

As demonstrated in our recent developmental study, each of the individual PL species has a characteristic profile of FA acyl chains that changes from birth until adulthood. On the other hand, all major PL exhibit some common features such as relatively higher proportions of OA and AA and lower proportions of LA and DHA in newborn rats than in adults. Another feature characteristic of the early postnatal period is the substantially increasing proportion of n-3 PUFA in all PL between delivery and the suckling-weaning transition (Novák et al. 2006). Several stress factors play a role in these developmental changes of the cardiac PL FA profiles, including nutritional, metabolic and hormonal changes as well as the increasing hemodynamic load (for review, see Novák et al. 2009).

In this study, we show for the first time the effect of pressure overload imposed on the rat heart just after birth, indicating additional n-3 PUFA increase superimposed upon that induced by normal postnatal growth. It has been reported that adult rat hearts subjected to pressure overload exhibit an increased proportion of DHA mainly in PE, counterbalanced by decrease of LA. Concerning PI, the proportion of DHA remained unchanged (Riebel et al. 1986). In our AC model, however, it was only PI in which the accumulation of DHA and DPA was the most pronounced at the expense of AA. It appears, moreover, that the effect on FA profile is more pronounced and selective when $\mathrm{AC}$ is imposed early after birth than in adulthood (Riebel et al. 1986).

Increased accumulation of n-3 PUFA in myocardial PL as a general adaptive response to various stressful conditions has been shown in several studies, for instance in rats exposed to chronic hypoxia (Ježková et al. 2002, Novák et al. 2004) and in animals chronically treated with catecholamines (Benediktsdottir et al. 1995, Gudbjarnason and Benediktsdottir 1996) or thyroid hormones (Hamplová et al. 2003). Noteworthy is that maturation of the $\beta$-adrenergic system and the increasing adrenergic responsiveness of the developing heart are 
under the control of thyroid hormones (Novotný et al. 1999). Hence, it seems conceivable that the hormonal changes mentioned above can contribute to the increased myocardial accumulation of n-3 PUFA associated with normal development and to the further potentiation of this effect by pressure overload.

In conclusion, accelerated cardiac growth stimulated by pressure overload imposed by AC soon after birth is not followed by proportional changes of membrane PL components. The attenuation of the PL increment after AC is accompanied by significant remodeling of the FA profile resulting in the selective accumulation of n-3 PUFA. These changes in the PL composition of membranes may affect the quality of lipid signaling molecules and properties of membrane proteins under stress conditions.

\section{Conflict of Interest}

There is no conflict of interest.

\section{Acknowledgements}

The authors thank J. Ševčíková and M. Pešková for excellent technical assistance. This work was supported by grants MSM 0021620858, AV0Z 50110509, SVV 265211 and PRVOUK - P25/LF1/2.

\section{References}

BENEDIKTSDOTTIR VE, SKULADOTTIR GV, GUDBJARNASON S: Effects of ageing and adrenergic stimulation on alpha 1- and beta-adrenoceptors and phospholipid fatty acids in rat heart. Eur J Pharmacol 289: 419-427, 1995.

CLUBB FJ, BISHOP SP: Formation of binucleated myocardial cells in the neonatal rat. An index for growth hypertrophy. Lab Invest 50: 571-577, 1984.

DINH DT, FRAUMAN AG, JOHNSTON CI, FABIANI ME: Angiotensin receptors: distribution, signalling and function. Clin Sci (Lond) 100: 481-492, 2001.

DORN GW, FORCE T: Protein kinase cascades in the regulation of cardiac hypertrophy. J Clin Invest 115: 527-537, 2005.

FOLCH J, LEES M, SLOAN-STANLEY GH: A simple method for the isolation and purification of total lipids from animal tissue. J Biol Chem 226: 497-509, 1957.

GIRARD J, FERRE P, PEGORIER JP, DUEE PH: Adaptations of glucose and fatty acid metabolism during perinatal period and suckling-weaning transition. Physiol Rev 72: 507-562, 1992.

GUDBJARNASON S, BENEDIKTSDOTTIR VE: Regulation of beta-adrenoceptor properties and the lipid milieu in heart muscle membranes during stress. Mol Cell Biochem 163-164: 137-143, 1996.

HAMPLOVÁ B, NOVÁKOVÁ O, TVRZICKÁ E, PELOUCH V, NOVÁK F: Effect of hypo- and hyperthyroid states on phospholipid composition in developing rat heart. Mol Cell Biochem 252: 295-303, 2003.

HAMPLOVÁ B, NOVÁKOVÁ O, TVRZICKÁ E, KOLÁŘ F, NOVÁK F: Protein kinase C activity and isoform expression during early postnatal development of rat myocardium. Cell Biochem Biophys 43: 105-117, 2005.

HAMPLOVÁ B, NOVÁK F, KOLÁŘ F, NOVÁKOVÁ O: Transient upregulation of protein kinase C in pressureoverloaded neonatal rat myocardium. Physiol Res 59: 25-33, 2010.

JEŽKOVÁ J, NOVÁKOVÁ O, KOLÁŘ F, TVRZICKÁ E, NECKÁŘ J, NOVÁK F: Chronic hypoxia alters fatty acid composition of phospholipids in right and left ventricular myocardium. Mol Cell Biochem 232: 49-56, 2002.

KOLÁŘ F, PAPOUŠEK F, PELOUCH V, OŠŤÁDAL B, RAKUŠAN K: Pressure overload induced in newborn rats: effects on left ventricular growth, morphology, and function. Pediatr Res 43: 521-526, 1998.

LI F, WANG X, CAPASSO JM, GERDES AM: Rapid transition of cardiac myocytes from hyperplasia to hypertrophy during postnatal development. J Mol Cell Cardiol 28: 1737-1746, 1996.

MRNKA L, NOVÁKOVÁ O, PELOUCH V, NOVÁK F: Phospholipid composition in the rat heart exposed to pressure overload from birth. Physiol Res 45: 83-85, 1996.

NOVÁKOVÁ O, DRNKOVÁ J, KUBIŠTA V, NOVÁK F: Regulation of phospholipid degradation and biosynthesis in the heart by isoprenaline: effect of mepacrine. Physiol Res 43: 151-156, 1994. 
NOVÁK F, TVRZICKÁ E, PELOUCH V, JEŽKOVÁ J, SMIK D, NOVÁKOVÁ O: Phospholipid composition of immature rat myocardium exposed to chronic hypoxia and the effect of normoxic recovery. Collect Czech Chem Commun 69: 674-688, 2004.

NOVÁK F, TVRZICKÁ E, HAMPLOVÁ B, KOLÁŘ F, NOVÁKOVÁ O: Postnatal development of phospholipids and their fatty acid profile in rat heart. Mol Cell Biochem 293: 23-33, 2006.

NOVÁK F, KOLÁŘ F, HAMPLOVÁ B, MRNKA L, PELOUCH V, OŠŤÁDAL B, NOVÁKOVÁ O: Myocardial phospholipid remodeling under different types of load imposed during early postnatal development. Physiol Res 58 (Suppl 2): S13-S32, 2009.

NOVOTNÝ J, BOUŘOVÁ L, MÁLKOVÁ O, SVOBODA P, KOLÁŘ F: G proteins, beta-adrenoreceptors and betaadrenergic responsiveness in immature and adult rat ventricular myocardium: influence of neonatal hypoand hyperthyroidism. J Mol Cell Cardiol 31: 761-772, 1999.

OLIVIERO PF, CHASSAGNE CF, KOLÁR̆ F, ADAMY CF, MAROTTE FF, SAMUEL JL, RAPPAPORT LF, OŠŤÁDAL B: Effect of pressure overload on angiotensin receptor expression in the rat heart during early postnatal life. J Mol Cell Cardiol 32: 1631-1645, 2000.

OŠTÁDAL B, OŠŤÁDALOVÁ I, DHALLA NS: Development of cardiac sensitivity to oxygen deficiency: comparative and ontogenetic aspects. Physiol Rev 79: 635-659, 1999.

PAGE E, MCCALLISTER LP: Quantitative electron microscopic description of heart muscle cells. Application to normal, hypertrophied and thyroxin-stimulated hearts. Am J Cardiol 31: 172-181, 1973.

REIBEL DK, O'ROURKE B, FOSTER KA, HUTCHINSON H, UBOH CE, KENT RL: Altered phospholipid metabolism in pressure-overload hypertrophied hearts. Am J Physiol 250: H1-H6, 1986.

ROUSER G, FLEISCHER S, YAMAMOTO A: Two dimensional thin layer chromatographic separation of polar lipids and determination of phospholipids by phosphorus analysis of spots. Lipids 5: 494-496, 1970.

SEDMERA D, THOMPSON RP, KOLÁŘ F: Effect of increased pressure loading on heart growth in neonatal rats. J Mol Cell Cardiol 35: 301-309, 2003.

TAPPIA PS: Phospholipid-mediated signaling systems as novel targets for treatment of heart disease. Can J Physiol Pharmacol 85: 25-41, 2007.

TVRZICKÁ E, VECKA M, STAŇKOVÁ B, ŽÁK A: Analysis of fatty acids in plasma lipoproteins by gas chromatography-flame ionisation detection. Quantitative aspects. Anal Chim Acta 465: 337-350, 2002. 